\title{
Phenotypic and Molecular Characterization of ESBL sproducing Enterobacteriaceaein A Tertiary Care Hospital
}

\author{
Dr.Savitha Rani ${ }^{1,}$ Dr.I.Jahnavi ${ }^{2,}$ Dr.K.Nagamani ${ }^{3}$ \\ Resident, Associate Professor, Professor Department ofMicrobiology Gandhi Medical College Hospital \\ SecunderabadTelangana.
}

\begin{abstract}
Background :There is an alarming magnitude of antibiotic resistance, production of Extended-Spectrum $\beta$ Lactamases (ESBLS) is a significant resistance-mechanism among Gram Negative bacteria posing challenge to the most coveted wellbeing of man. The Present study was undertaken to detect the prevalence of ESBLs producers, their pedantic antibiogram, and their genes, bla CTX-M, bla TEM \&bla SHV by Multiplex PCR.

Methodology:In this prospective study, 240 non repetitive Isolates of Enterobacteriaceae from various samples of different departments of Gandhi Hospital were processed for the presence of ESBL by Phenotypic screening, Jarlier DDST, Phenotypic confirmatory test (CLSI guidelines),Vitek2 System, Multiplex PCR for Molecular characterization over a period of 11/2 years in the Department of Microbiology Gandhi Medical College and Hospital,Secunderabad,Telangana.

Results: Out of 240 Gram negative isolates $35.8 \%$ were phenotypically confirmed ESBLs producers. OP samples yielded 49/86(56.98\%), whereas IP $37 / 86(43.02 \%)(P$ value 0.0327 statistically significant).Medical and Surgical ICUs yielded 50\%, Predominant organism isolated was E.coli 61.62\%. All the isolates were 100\% resistant to $3^{\text {rd }}$ generation Cephalosporins, 100\% Sensitive to Carbapenems, Genotypic characterization was detected in 78 Isolates. Single gene was found in 73.08\%. Single Predominant gene was blaCTX-M 71.9\%, commonest multiple genes combination was CTX-M\&SHV12.82\%.

Conclusion:Our study confirms global trend of resistance to beta-lactam antibiotics. ESBL strains are usually multi-drug resistant.An appropriate and judicious antibiotic use may lead to withdrawal of the selective pressure.
\end{abstract}

Keywords: ESBLs, DDST, PCT, VITEK 2, CTX-M, TEM, SHV

\section{Introduction}

The rapid and irrepressible increase in antimicrobial resistance more so the ESBLs production by Enterobacteriaceae was a worrying global health concern, posing unique challenges to clinical microbiologists, clinicians, infection control professionals and antibacterial-discovery scientists. $\beta$-Lactams are safest and most frequently prescribed antimicrobial agents all over the world in treating Gram positive and Gram negative infections ${ }^{1}$.

Betalactamases were numerous and the genes encoding them mutate continuously in response to heavy pressure of antibiotic use.They have spread threateningly in many regions of the world creating serious problem to the currently available antibiotic armory.Being Plasmid mediated, it facilitated the dissemination of resistance not only to $\beta$ lactams but also to other commonly used antibiotics such as fluoroquinolones and aminoglycosides. ${ }^{2}$ Quinolone antibiotics were potent promoters of ESBL transmission.Prevalence of ESBLsin United Statesamong E. coli ranges from 0 to $25 \%$ (NNIS) ${ }^{3}$.In Japan $<0.1 \%^{4}$, in Korea $4.8 \%$, in Taiwan $8.5 \%$ and up to $12 \%$ in Hong Kong. ${ }^{5}$ In India various authors reported the prevalence of ESBLs ranging from $4 \%$ to $83 \%{ }^{6}$

The first beta-lactamase TEM a plasmid-mediated enzyme named after the patient Temnonierafrom whom it was discovered in Athens of Greece in 1960s,now 175 different TEM enzymes and 127 SHV(Sulfhydryl variable) enzymes exist (www.lahey.org/studies/). Up to $20 \%$ of the ampicillin resistance in K pneumoniawas due to SHV-1. In 1989Bauernfeindet al from Germany reported CTX-M-1(Cefotaximase). It had spread globally,currently more than 80 CTX-M enzymes were known.The initial CTX-M arose in the nosocomial setting and spread to the community.The reporting of ESBL producers help the clinicians to select the appropriate antibiotics for the treatment and to take proper precautions to prevent the spread. Some ESBLs may not reach a level to be detected by disk diffusion, but result in treatment failure,identifying the exact ESBL subtype was possible only by molecular detection methods. Though so many types of ESBLs exist majority of the ESBLs were derivatives of TEM, SHV or CTX-M enzymes. Judicious usage of extended-spectrum cephalosporins, periodic surveillance of antibiotic resistance patterns for formulating Antibiotic Policy, and efforts to decrease empirical antibiotic therapy prevent spread and outbreaks of ESBL producing bacteria 


\section{Aims}

1)To determine the prevalence of extended spectrum beta lactamases (ESBLs) among clinical isolates of Enterobactereaceae. 2) To determine their pedantic antibiogram, and Minimum Inhibitory Concentration (MIC) of Antibiotics by Vitek 2 System,3)To detect main ESBL encoding genes such as CTX-M, SHV and TEM by Multiplex PCR (M-PCR) in Gandhi hospital, Telangana.

\section{Materials And Methods}

A total of 240 non repetitive, clinical isolates of Enterobacteriaceae from a variety of clinical samples from Out-patients and In-patients of Gandhi Hospital were processed in the Department of Microbiology laboratory over a period of $1 \frac{1}{1 / 2}$ years (April 2013 - September 2014) The Isolates were identified, both by conventional Method (Mackie and McCartney Practical Microbiology ${ }^{8}$, Koneman`s color atlasand textbook of diagnostic microbiology Diagnostic microbiology ${ }^{9}$ ) and Vitek 2 System [using Identification GN13 cards; AST (Antibiotic Susceptibility Testing) cards bio Merieux, Durham, NC]

The Isolates were screened for possible ESBL production by Screening tests, Doubledisc synergy test (DDST) and the Phenotypic Confirmatory Test (PCT) as per the guidelines issued by Clinical Laboratory standards Institute 2013. ${ }^{10}$

Co-resistance to fluoro-quinolones, aminoglycosides, trimethoprim-sulphamethoxazole, and beta lactamase inhibitor combinations and susceptibility to carbapanems were determined by disc diffusion method, control strains used were

Positive control : ESBL-producing organism (KlebsiellapneumoniaeATCC 700603)

Negative control: Non-ESBL-producing organism (Escherichia coli ATCC 25922)

The 86 ESBL producers confirmed by PCT were subjected for detection of genes encoding Beta Lactamases by multiplex PCR amplification using primer sets for blaCTX-M, blaTEM, blaSHV genes, according to the methodology described in the Sambrook and Russell ${ }^{11}$ and referred in an article by Meeta Sharma et $\mathrm{al}^{12}(2012)$ for primers. Molecular Method: A)DNA extractionwas done by boilingethod,B)DNAamplification-Multiplex PCRC) Gel electrophoresisand Visualization of PCR products under UV lights by trans-illuminator. The Master mixwas prepared as follows: $2.5 \mu \mathrm{L}$ of PCR buffer, $2.5 \mu \mathrm{Lof} 10 \mathrm{mM}$ dNTP mix, $1 \mu \mathrm{L}$ of Taq DNA Polymerase, $22 \mu \mathrm{L}$ of RNase free water and $2 \mu \mathrm{L}$ of each of the forward and reverse primers.

CTX-M beta-lactamase: $560 \mathrm{bp}$

Forward Primer : 5'- GAAGGTCATCAAGAAGGTGCG -3'

Reverse primer : $5^{\prime}$ - GCATTGCCACGCTTTTCATAG- 3

TEM beta-lactamase: $459 \mathrm{bp}$

Forward Primer : 5' - GAGACAATAACCCTGGTAAAT- 3

Revers primer : $5^{\prime}-$ AGAAGTAAGTTGGCAGCAGTG- $3^{\prime}$

SHV beta-lactamase: $383 \mathrm{bp}$

Forward Primer : 5'-GTCAGCGAAAAACACCTTGCC- 3'

Revers primer :5'-GTCTTA`TCGGCGATAAACCAG- 3'

DNA amplification in thermal cycler: An initial denaturation step at $95^{\circ} \mathrm{C}$ for $5 \mathrm{~min}$ followed by 35 cycles of denaturation at $95^{\circ} \mathrm{c}$ for $30 \mathrm{sec}$, annealing at $60^{\circ} \mathrm{c}$ for $30 \mathrm{sec}$, extension at $72^{\circ} \mathrm{c}$ for 2 min ending with a final extension step at $72^{\circ} \mathrm{C}$ for $10 \mathrm{~min}$ and followed by a hold at $4^{\circ} \mathrm{C}$.

Agarose gel electrophoresis:Was done for The PCR products with 1.8\% agarose gel using suitable molecular weight markers.

\section{DDST PCT1)Gel documentation2)Gel documentation}

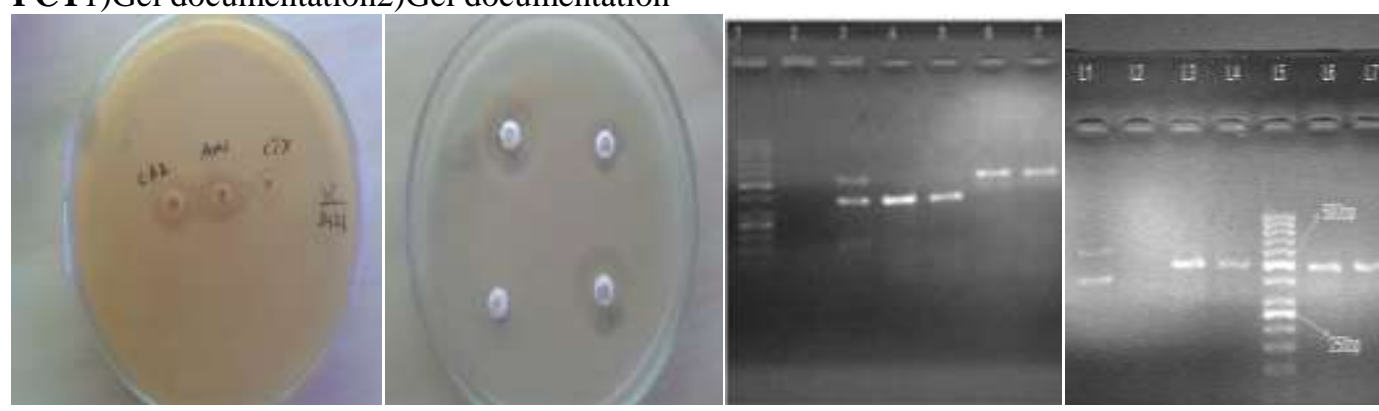

1)Gel documentation of Multiplex PCR analysis showing 2)Gel documentation of Multiplex PCR

Lane-1 ,50bp DNA ladder,Lane 2 Negative controlanalysisthe presence of ESBL genes. 
Lane3positive control SHV,CTX-M Showing the presence of ESBL genesLane-1 Positive Control, Lane 2 Negative Control

Lane -4 and 5-SHV ,Lane -6 and 7 CTX-M Lane -3 ,4,6,and7 TEM, Lane 5 DNA ladder50 bp

EPI INFO 2000 version statistical soft ware package was used for statistical analysis. Chi-Square test was applied to test whether differences between values were significant. $\mathrm{P}$ values $<0.05$ was considered as statistically significant. $\mathrm{P}>0.05$ insignificant.

\section{Results}

Out of 240 Gram Negative isolates, 35.83\% were ESBLs producers by PCT and by Vitek 2 system, molecular characterization was found among 32.5\%. More number of Isolates were $42.1 \%$ among 41-60 years of age group, followed by $>60 y$ rs $(36.5 \%), 21-40$ ( 26.9\%) and 1-20( $18.18 \%)$. Males were 38.83\%, Females $33.5 \%$ (Table1).More number of isolates were fromOP49/86(56.98\%) whereas IP 37/86 (43.02\%)(Table2).p value 0.0327 statistically significant. Medical and Surgical ICUs yielded 50\% followed by ICCU 47\%, Ortho 45\%, OBG 25\%, Surgery 20\%, Medicine $16.66 \%$ and least from ENT 6.6\%(Table 3).Higher frequency among Pus samples $41.8 \%$ followed by Urine $36.25 \%$, Sputum $33.3 \%$ lowest from blood $15 \%$ (Table 4). Escherichia coliwas the most common isolate $61.63 \%$ followed by klebsiella pneumonia30.23\%, Proteus species $5.81 \%$, Citrobacter 2.33\%. All the isolates were Resistant (100\%)toCeftazidime MIC >=4, Cefotaxime, Ceftrioxone MIC $>=8$, Cefuroxime MIC $>=64$ andAztreonum $\mathrm{MIC}>=2$, Sensitive (100\%) to Imipenem, Meropenem, Doripenem and Ertapenem MIC rangewas $<=0.12$ to $<0.5$.

Among theE.coli isolates highest resistance was observed with Nalidixic acid $92.45 \%$,followedby Amoxicillin/clavulanicacid 88.67\%, Trimethoprim/Sulfmethaxazole84.9\%, Ciprofloxacin 81.13\%, Levofloxacin $69.81 \%$,Minocycline $67.92 \%$ and sensitivity was high with Cefperazone/sulbactam96.23\% followed by Piperacillin/Tazobactam79.24\%.Most prevalent gene was CTX-M $60.37 \%$ followed by TEM11.32\%,SHV3.77\%,CTX-M+TEM9.4\%,CTX-M+ SHV11.32\%, not characterized were $3.77 \%$.

Among the Klebsiella isolates resistance was highest 92.3\%, with Amoxicillin/clavulanic acid and Ciprofloxacin followed byNalidixic acid $88.46 \%$, Trimethoprim /sulfmethaxazole $76.92 \%$, Levofloxacin $76.9 \%$ and highest sensitivity was observed with Cefperazone/sulbactam $96.16 \%$ followed by Gentamycin 69.24\%Piperacillin/ Tazobactam 65.39\%,Amikacin61.54\%(Table 5).Out of 86 phenotypically confirmed ESBLs 78 were genotypicallyconfirmed(Table 6).

Single gene was found in $73.08 \%$, multiple genes were found in $26.92 \%$ (Table 7). Most prevalent gene was CTX-M 34.61\%, followed by TEM 15.38\%, SHV11.53\%, multiple genes were TEM+ SHV 19.23\%,CTXM+SHV15.38\%, not characterized was $3.84 \%$.

Proteus species(20\%), and Citrobacter(8\%) were isolated from surgery department.Among the Proteus SHV $(20 \%)$ and TEM+SHV(20\%), $60 \%$ were not characterized, Citrobacter isolates $100 \%$ were not characterized(Table 8).

\section{Discussion}

The emergence of ESBL-producing bacteria had been strikingly increasing worldwide, and still considered as threat since they were transferred by plasmid which can be easily transmitted between the species. ${ }^{13}$ The therapeutic options for these infections became increasingly narrowed down of the pipeline increasing morbidity and mortality.Hence continuous monitoring systems and effective infection control measures were absolutely necessary.

The present study revealed $35.8 \%$ prevalence of ESBL producers which correlated with $\mathrm{N}$ Fam et al ${ }^{13}$ from Egypt $35.7 \%$, slightly lesser than NeemaShashwatiet al ${ }^{14}$ from Mumbai $48.27 \%$, Meeta Sharma ${ }^{12}$ from Jaipur reported $52.49 \%$, Rodrigues $C$ et al $^{15}$ fromMumbai showed 53\%whereas R.Canton et al ${ }^{16}$ from Europe and Goosens et $\mathrm{al}^{17}$ found $1-12 \%$ to as high as $39-47 \%$ in Russia, Poland and Turkey.The wide variation in the prevalence was probably due to differences in the risk factors and the excessive use of cephalosporins, and a high rate of patient transfer from the peripheral centers who received prior multiple antimicrobial treatments with high end antibiotics Mathur $\mathrm{P}$ et $\mathrm{l}^{18}$

The study showed high prevalence of the ESBL producers among 41-60 years age group (46.51\%) which was correlating with MetriBasvaraj et $\mathrm{al}^{19} 41-60 \mathrm{yrs}$, Lautenbach et $\mathrm{al}^{20} 54 \mathrm{yrs}$ median age, This may be because more number of patients were hospitalized in that age group and had other risk factors like Diabetes Mellitus. Whereas GopalKashyapet $\mathrm{al}^{21}$ showed20-40yrs age group (43\%)as UTI was more common in middle age group.

There was slight male preponderance $38.83 \%$ this was correlating with Lautenbach et al, ${ }^{20} \mathrm{Nema}$ Shashwati, ${ }^{14}$ Nibedita Das et al. ${ }^{22}$ Whereas femalepreponderance was noticed byKiratisin et al $^{23}$ from Thailand and MetriBasvarajet $\mathrm{al}^{19}$ from Bijapur as number of female patients suffering from UTI with ESBL producers were more. 
More ESBL isolates were from Out-Patient departments of various disciplines 49/86(56.98\%) whereas IP 37/86 (43.02\%) (P value 0.0327 statistically significant.), which was in accordance withShakti Rath et $\mathrm{al}^{24}$ from Bhubaneswar OP $56.31 \%$, IP $43.69 \%$, slightly lower than GopalKashyap et $\mathrm{al}^{21}$ OP $65.6 \%$ Whereas VemulaS et $\mathrm{al}^{25}$ from Kurnool, reported more from IP departments $60 \%$. In the beginning ESBLs were a hospital based crisis but now becoming more common among community acquired isolates, this should alert the physician.Medical and surgical ICUs yielded more number of isolates $50 \%$, followed by the ICCU $45 \%$ this was comparable withreports by Varsha Gupta et $\mathrm{al}^{26}$ from Chandigarh and FaustineNdugulile et $\mathrm{al}^{27}$ from Tanzania, Marra ${ }^{28}$ et al $^{2}$ from Brazil., and Mathur et ${ }^{18}$ al $79 \%$ from AIIMS New Delhi, whereas MethriBasavarajuet al ${ }^{19}$ showed more from ICCU.This could be due to the prolonged hospital stay, indwellingCanulas, catheters, endotracheal or nasogastric tubes, gastrostomies or tracheostomies and due to severe illness.

ESBL distribution was more among pus samples 41.8\%, which was correlating with Dasgupta Rubin et $\mathrm{al}^{29}$ from Dehradun, ManinderKaur et $\mathrm{al}^{30}$ fromAmritsar andVarsha Gupta et $\mathrm{al}^{26}$ from Chandigarh Whereas GauravDalela et $\mathrm{al}^{31}$ and R.Eshwar Singh et $\mathrm{al}^{32}$ reported urine and Marra ${ }^{28}$ from blood.Majorityof ESBL positive isolates were E. coli $61.6 \%$ this wasslightly lesser than that ofR.Eshwar Singh et $\mathrm{al}^{32} 67 \%$ from Devangere,Pathak $\mathrm{A}^{33}$ from Ujjain $69 \%$, in a reportfrom Pondicherry $81 \%$ Slightly higher compared to NemaShashwati et $\mathrm{al}^{16} 50.14 \%$.Rudresh et $\mathrm{al}^{34} 40.2 \%$. The percentage of Klebsiellapneumoniae $30.2 \%$, was in accordance with M.Karagaret $\mathrm{al}^{35} 30.55 \%$ from Iran.The percentage of Proteus spp $5.81 \%$ was correlating withVidyaPaiet $\mathrm{al}^{36} 5.5 \%$ from Mangalore. The percentage of Citrobacterspp $2.32 \%$ was less comparedChoiSet $\mathrm{al}^{37}$ who reported $4.9 \%$ from Korea, very much less compared to Shresthaetal ${ }^{38} 16.6 \%$ fromNepal.

Of the 240 isolates ESBL producers by using the PCT were 86(100\%), DDST $45.34 \%$. Emery ${ }^{39}$ also found DDST could not detect all ESBL-producing isolates. PCT was technically simple cost effective, and $100 \%$ in concordance with MIC of Vitek 2 System report.BradfordPA ${ }^{1}$ explained that false-positives may occur if the isolate lacks ESBL but produces an excess of TEM-1 or SHV-1on the other hand, isolates harboring both ESBLs and AmpC-type $\beta$-lactamases may result in false-negative results.

VITEK 2 system a reliable timesaving tool for routine identification of ESBL-producing strains. All the isolates 86 were sensitive $100 \%$ to Carbapenamsasthese antibiotics were highly stable to $\beta$-lactamase activity they remain the only choice of treatment for infections by ESBLs producers.All the Isolates were $100 \%$ resistant to $3^{\text {rd }}$ generation Cephalosporins\&Aztreonum, which was in accordance with ManoharanAet $\mathrm{al}^{40}$, from Vellore,Sanjeev Kumar et $\mathrm{al}^{41}$ from Udaipur, Akbar M. Rafay et $\mathrm{a}^{42}$ from Oman. R.Bonnetet al ${ }^{43} \mathrm{Nema}^{4}$ Shashwati ${ }^{16}$ found Most of them were ceftazidimases, and only a few are cefotaximases. This high degree of drug resistance could be due to the availability of drugs to patients with or without any prescription and discontinuing them in the middle of the course.

E.coli isolates showed highest resistance toNalidixic acid $92.45 \%$, followed by Amoxicillin/clavulanicacid $\quad 88.67 \% \quad$ Trimethoprim/sulfmethaxazole $84.9 \%, \quad$ Ciprofloxacin 81.13\%,Levofloxacin69.81\%,Minocycline 67.92\%, Nitrofurantoin $60 \%$ and sensitivity was $100 \%$ for Carbapenam,96.23\% for Cefperazone/sulbactam followed by Piperacillin/Tazobactam83.02\% which was nearer to the reports of MeetaSarma et al from Jaipur, ${ }^{12}$ Wong-Beringer ${ }^{44}$ et al.from Pakistan.The Klebsiella isolates were highly resistant to Amoxicillin\&clavulanicacid and Ciprofloxacin92.3\% followed byNalidixic acid $88.46 \%$ Trimethoprim\&sulfmethaxozole, Levofloxacin 76.92\%, sensitive was $100 \%$ for Carbapenam $96.16 \%$ for Cefperazone/sulbactamfollowed byGentamycin 69.24\%, Piperacillin/ Tazobactam 65.39\%, Amikacin 61.54\%.. Which was near toMeetha Sharma ${ }^{12}$ from Jaipur, Ullah et a $l^{45}$ from Pakistan

Jacoby $^{46}$ explained, that this may be due to the occurrence of genes encoding resistance to aminoglycoside, trimethoprim-Sulfomethoxazole, and quinolones on the same plasmid that encodes for ESBL production. Martínez-Martínez ${ }^{47}$ and colleagues in their analysis found porin loss among the ESBL producing K.pneumoniae also showed active efflux of quinolones, Energy dependent accumulation of Norfloxacin.

Out of 86 phenotypically confirmed ESBLs 78 showed genotypic characterization. More number of isolates expressed single genes $73.71 \%$ multiple genes were $26.29 \%$. This was nearly correlating withMeeta Sharma ${ }^{12}$ from Jaipur, Mehdi Kargar ${ }^{35}$ from Iran. Whereas Kaftandzievaet $\mathrm{al}^{48}$ from Skopze reported mixed genes.

Among the ESBLs Producing E.coliCTX-M-60.37\% TEM-11.32\% SHV-3.77\% CTX-M+TEM 9.4\%, CTX-M+SHV $11.32 \%$, isolatesnot characterizedwere $3.77 \%$ nearly correlating withManinderKaur ${ }^{27} \mathrm{CTX}-\mathrm{M}$ genes 59.32\%, CTX-M+TEM 8.4\%. Meeta Sharma et $\mathrm{al}^{12}$ from Jaipur CTX-M80\%,Ho.P.L ${ }^{49}$ observed UTI due to CTX-M ESBL produce E. coli strains, A Manoharanet al ${ }^{40}$ observed TEM and CTX-M were predominant. Most prevalent gene among Klebsiella was CTX-M 34.61\%, followed by TEM+ SHV 19.23\%, TEM 15.38, CTX-M + SHV15.38\%, SHV11.53\%, not characterizedwas3.84\%. Slightly lesser thanFeizabadi MMet al ${ }^{50}$ reported bla(CTX-M-I) $46.51 \%$. Mubarak et $\mathrm{al}^{51}$ from UAE reported the emergence and dissemination of CTXM-15 as87\%among E. coli and K. pneumoniae CTX-M enzymes are now endemic over a wide geographic area, Most strains producing CTX-M enzymes seem to be implicated in nosocomial infections.

James H. Jorgensen ${ }^{52}$ found CTX-M prevalent globally Livermore et al $^{53}$ R.Bonnet ${ }^{54}$ found CTX-M

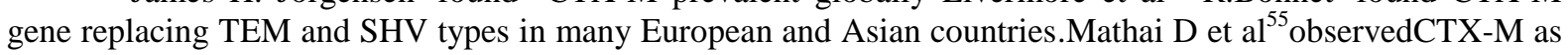


Predominant gene in India. The easy dissemination, extraordinary spread of the CTX-M enzymes deserving an uncontrolled pandemic scenario was due to the resistance determinants and the frequency of transfer in the range of $10^{-7}$ to $10^{-2}$ per donor cellbla $a_{\mathrm{CTX}-\mathrm{M}}$ harboring plasmids.

Table No.1 Sex wise distribution of ESBL producers

\begin{tabular}{|l|l|l|l|}
\hline Sex & $\begin{array}{l}\text { Total } \\
\mathrm{n}=240\end{array}$ & $\begin{array}{l}\text { ESBL Positive } \\
(\mathrm{n}=86)\end{array}$ & $\begin{array}{l}\text { ESBL } \\
\text { Positive } \\
\%\end{array}$ \\
\hline Male & 103 & 40 & 38.8 \\
\hline Female & 137 & 46 & 33.5 \\
\hline
\end{tabular}

Table No.2 Distribution of ESBL producers in O P and I P Patients

\begin{tabular}{|l|l|l|l|c|}
\hline $\begin{array}{l}\text { Total no } \\
\mathrm{N}=240\end{array}$ & \multicolumn{2}{|l|}{$\begin{array}{l}\text { Total I.P } \\
\mathrm{N}=136\end{array}$} & \multicolumn{2}{l|}{$\begin{array}{l}\text { Total O.P } \\
\mathrm{N}=104\end{array}$} \\
\hline $\begin{array}{l}\text { Total ESBL positive } \\
\mathrm{N}=86\end{array}$ & $\begin{array}{l}\text { ESBL positive } \\
\mathrm{N}=37\end{array}$ & $\begin{array}{l}\text { ESBL } \\
\text { negative } \\
\mathrm{N}=99\end{array}$ & $\begin{array}{l}\text { ESBL } \\
\text { positive } \\
\mathrm{N}=49\end{array}$ & $\begin{array}{l}\text { ESBL } \\
\text { negative } \\
\mathrm{N}=55\end{array}$ \\
& & & \\
\hline
\end{tabular}

Table No.3Distibution of ESBL Producers among various Departments

\begin{tabular}{|l|l|l|l|l|l|l|}
\hline $\begin{array}{l}\text { Departme } \\
\text { nt }\end{array}$ & $\begin{array}{l}\text { Tot } \\
\text { al } \\
\text { Iso } \\
\text { late } \\
\text { s }\end{array}$ & $\begin{array}{l}\text { Totalno } \\
86\end{array}$ & $\begin{array}{l}\text { Escherich } \\
\text { ia coli } \\
\mathrm{n}=53\end{array}$ & $\begin{array}{l}\text { Klebsiella } \\
\text { pneumonia } \\
\text { e. } \\
\mathrm{n}=26\end{array}$ & $\begin{array}{l}\text { Proteus } \\
\text { SPP } \\
\mathrm{n}=5\end{array}$ & $\begin{array}{l}\text { Citrobacter } \\
\text { Freundii. } \\
\mathrm{n}=2\end{array}$ \\
\hline MICU & 40 & $20(50 \%)$ & $15(28.3 \%)$ & $5(19.23 \%)$ & - & - \\
\hline SICU & 50 & $25(50 \%)$ & $12(22.6 \%)$ & $6(23.07 \%)$ & $\begin{array}{l}5 \\
(20 \%)\end{array}$ & $2(8 \%)$ \\
\hline ICCU & 32 & $15(47 \%)$ & $11(20.7 \%)$ & $4(15.3 \%)$ & - & - \\
\hline Ortho & 20 & $9(45 \%)$ & $4(7.54 \%)$ & $5(19.23 \%)$ & & \\
\hline OBG & 25 & $5(20 \%)$ & $3(5.6 \%)$ & $2(7.69 \%)$ & - & - \\
\hline Medicine & 25 & $5(20 \%)$ & $3(5.6 \%)$ & $2(7.69 \%)$ & - & - \\
\hline Surgery & 25 & $5(20 \%)$ & $4(7.54 \%)$ & $1(3.84 \%)$ & - & - \\
\hline ENT & 30 & $2(6.6 \%)$ & $1(1.88 \%)$ & $1(3.84 \%)$ & - & - \\
\hline
\end{tabular}

Table No 4Distributionof ESBL producing organism in various clinical samples

\begin{tabular}{|l|l|l|l|l|l|l|}
\hline Name of the organism & Pus & Urine & Sputum & Stool & $\begin{array}{l}\text { Vaginal } \\
\text { swabs }\end{array}$ & Blood \\
\hline Escherichia coli $\mathrm{n}=53$ & $28(52.8)$ & $21(39.62 \%)$ & - & $2(3.77 \%)$ & $2(3.77 \%)$ & \\
\hline Klelpneumoniae. $\mathrm{n}=26$ & $11(42.3)$ & $8(30.76 \%)$ & $4(15.38 \%)$ & & & $\begin{array}{l}3(11.5 \\
\%)\end{array}$ \\
\hline ProteusSppn=5 & $5(100 \%)$. & & & & & \\
\hline $\begin{array}{l}\text { Citrobacterfreundii. } \\
\mathrm{n}=2\end{array}$ & $2(100 \%)$. & & & & & \\
\hline
\end{tabular}

Table No.5Antimicrobial resistance pattern among ESBL positive isolates

\begin{tabular}{|c|c|c|c|c|c|c|c|c|c|c|c|c|}
\hline \multirow[t]{2}{*}{$\begin{array}{l}\text { Name of } \\
\text { Antibiotic }\end{array}$} & \multicolumn{3}{|c|}{$\begin{array}{l}\text { Escherichia coli, } \\
\mathrm{N}=53\end{array}$} & \multicolumn{3}{|c|}{$\begin{array}{l}\text { Klebsiellapneumonia } \\
e \\
N=26\end{array}$} & \multicolumn{3}{|c|}{$\begin{array}{l}\text { Proteus spp. } \\
N=5\end{array}$} & \multicolumn{3}{|c|}{$\begin{array}{l}\text { Citrobacterspp } \\
\mathrm{N}=2\end{array}$} \\
\hline & $\mathrm{S}$ & $\mathrm{I}$ & $\mathrm{R}$ & $S$ & $I$ & $R$ & $S$ & $I$ & $R$ & $\mathrm{~S}$ & I & $\mathrm{R}$ \\
\hline Ceftazidime & - & - & 53 & - & - & 26 & - & - & 5 & - & - & 2 \\
\hline Ceftriaxone & - & - & 53 & - & - & 26 & - & - & 5 & - & - & 2 \\
\hline Cefuroxime & - & - & 53 & - & - & 26 & - & - & 5 & - & - & 2 \\
\hline
\end{tabular}




\begin{tabular}{|c|c|c|c|c|c|c|c|c|c|c|c|c|}
\hline $\begin{array}{l}\text { Cefuroxime } \\
\text { axetil }\end{array}$ & - & - & 53 & - & - & 26 & - & - & 5 & - & - & 2 \\
\hline Ampicillin & - & - & 53 & - & - & 26 & - & - & 5 & - & - & 2 \\
\hline Amoxiclav & 4 & 2 & 47 & 1 & 1 & 24 & 0 & 0 & 5 & 0 & 0 & 2 \\
\hline Nalidixic acid & 4 & - & 49 & 3 & - & 23 & & & 5 & & & 2 \\
\hline $\begin{array}{l}\text { Trimethoprim/ } \\
\text { sulfamethoxaz } \\
\text { ole }\end{array}$ & 8 & - & 45 & 6 & - & 20 & - & - & 5 & - & - & 2 \\
\hline Ciprofloxacin & 10 & - & 43 & 2 & & 24 & 2 & - & 3 & 1 & - & 1 \\
\hline Levofloxacin & 14 & 2 & 37 & 4 & 2 & 20 & 3 & - & 2 & 1 & - & 1 \\
\hline Minocycline & 16 & 1 & 36 & 11 & 2 & 13 & - & - & 5 & - & - & 2 \\
\hline Nitrofurantoin & 26 & - & 27 & 11 & - & 15 & - & - & 5 & - & - & 2 \\
\hline Gentamicin & 25 & 1 & 27 & 17 & 1 & 8 & 2 & - & 3 & - & - & 2 \\
\hline Tigecycline & 31 & - & 22 & 11 & - & 15 & - & - & 5 & - & - & 2 \\
\hline Amikacin & 33 & - & 20 & 16 & - & 10 & 3 & - & 2 & 1 & - & 1 \\
\hline $\begin{array}{l}\text { Piperacillin } \\
\text { /Tazobactum }\end{array}$ & 42 & 2 & 9 & 16 & 1 & 9 & 1 & 1 & 3 & 1 & - & 1 \\
\hline $\begin{array}{l}\text { Cefperazone/s } \\
\text { ulbactum }\end{array}$ & 50 & 1 & 2 & 15 & 1 & 10 & 3 & 1 & 1 & 2 & 0 & \\
\hline Imipenem & 53 & - & - & 26 & - & - & 5 & - & - & 2 & - & - \\
\hline Doripenem & 53 & - & - & 26 & - & - & 5 & - & - & 2 & - & - \\
\hline Meropenem & 53 & - & - & 26 & - & - & 5 & - & - & 2 & - & - \\
\hline Ertapenem & 53 & - & - & 26 & - & - & 5 & - & - & 2 & & -- \\
\hline
\end{tabular}

Table No 6 Phenotypic and Genotypic distribution of ESBLs

\begin{tabular}{|l|l|l|l|}
\hline Total no of samples & $\begin{array}{l}\text { Phenotypic confirmatory test } \\
\text { positives }\end{array}$ & $\begin{array}{l}\text { Genotype } \\
\text { Negative }\end{array}$ \\
\hline 240 & 86 & $78(90.69 \%)$ & $8(9.3 \%)$ \\
\hline
\end{tabular}

Table No. 7 Distribution pattern of Single and Multiple genes among ESBL producers

\begin{tabular}{|l|l|l|l|}
\hline Phenotypic ESBL positives & Genotypic ESBL positives & Single genes & $\begin{array}{l}\text { Multiple } \\
\text { genes }\end{array}$ \\
\hline 86 & $78(90.69 \%)$ & $57(73.07 \%)$ & $21(26.92 \%)$ \\
\hline
\end{tabular}

Table No 8 Distribution of ESBL genes among the different isolates

\begin{tabular}{|c|c|c|c|c|c|c|c|c|}
\hline Organism & $\begin{array}{l}\text { CTX-M } \\
\mathrm{N}=41\end{array}$ & $\begin{array}{l}\text { TEM } \\
\mathrm{N}=10\end{array}$ & $\begin{array}{l}\mathrm{SHV} \\
\mathrm{N}=6\end{array}$ & $\begin{array}{l}\text { CTXM+ } \\
\text { TEM } \\
\mathrm{N}=5\end{array}$ & $\begin{array}{l}\text { CTX-M + } \\
\text { SHV } \\
\mathrm{N}=10\end{array}$ & $\begin{array}{l}\text { TEM+ } \\
\text { SHV } \\
6\end{array}$ & $\begin{array}{l}\text { Not } \\
\text { character } \\
\text { ized } \\
\text { n-8 }\end{array}$ & Total \\
\hline E.coli & $\begin{array}{l}32 \\
60.37 \%\end{array}$ & $\begin{array}{l}6 \\
11.32 \%\end{array}$ & $\begin{array}{l}2 \\
3.77 \%\end{array}$ & $\begin{array}{l}5 \\
9.4 \%\end{array}$ & $\begin{array}{l}6 \\
11.32 \%\end{array}$ & - & $\begin{array}{l}2 \\
3.77 \%\end{array}$ & 53 \\
\hline $\begin{array}{l}\text { K.pneumo } \\
\text { niae }\end{array}$ & $\begin{array}{l}9 \\
34.61 \% \\
\end{array}$ & $\begin{array}{l}4 \\
15.38 \%\end{array}$ & $\begin{array}{l}31.53 \% \\
\end{array}$ & - & $\begin{array}{l}4 \\
15.38 \%\end{array}$ & $\begin{array}{l}5 \\
19.23 \%\end{array}$ & $\begin{array}{l}1 \\
3.84 \% \\
\end{array}$ & 26 \\
\hline $\begin{array}{l}\text { Proteus } \\
\text { spp }\end{array}$ & - & - & $\begin{array}{l}1 \\
20 \% \\
\end{array}$ & - & - & $\begin{array}{l}1 \\
20 \%\end{array}$ & $\begin{array}{l}3 \\
60 \% \\
\end{array}$ & 5 \\
\hline $\begin{array}{l}\text { Citribacte } \\
\mathrm{r}\end{array}$ & - & - & - & - & - & - & $\begin{array}{l}2 \\
100 \% \\
\end{array}$ & 2 \\
\hline
\end{tabular}




\section{Conclusion}

ESBL strains are usually multi-drug resistant. Quick detection of these strains in microbiology laboratories is very important,A combination of different tests can be useful for accurate identification so that appropriate treatment is instituted to reduce morbidity and mortality substantially. Data on institutional antibiograms are necessary for formulating antibiotic policy and for Antibiotic Stewardship to control Hospital Acquired Infections(HAI). An appropriate and judicious antibiotic use may lead to withdrawal of the selective pressure.

\section{References}

[1]. Bradford P.A. Extended-spectrum beta-lactamases in the 21st century: characterization, epidemiology, and detection of this important resistance threat. Clin.Microbiol. Rev. 2001;14:933-951

[2]. Pfaller MA, Segreti J. Overview of the epidemiological profile and laboratory detection of extended-spectrum beta-lactamases. Clin Infect Dis. 2006 Apr 15;42Suppl 4:S153-63.

[3]. National Nosocomial Infections Surveillance (NNIS) System Report, data summary from January 1992 through June 2004 issued October 2004. Am J Infect Control. 2004;32(8):470-485.

[4]. Yagi T, Kruokawa H, Shibata N, et al. A preliminary survey of extended-spectrum $\beta$-lactamases (ESBLs) in clinical isolates of Klebsiellapneumoniae and Escherichia coli in Japan. FEMS Microbiol Lett.2000;184:53-56. [PubMed]

[5]. Yan JJ, Wu SM, Tsai SH, Wu JJ, Su IJ.Prevalence of SHV-12 among clinical isolates of Klebsiellapneumoniae producing extended-spectrum $\beta$-lactamases and identification of a novel AmpC enzyme (CMY-8) in southern Taiwan.Antimicrob Agents Chemother. 2000;44:1438-1442. [PMC free article][PubMed]

[6]. Hansotia JB, Agarwal V, Pathak AA, Saoji AM. Extended spectrum beta-lactamase mediated resistance to third generation cephalosporins in Klebsiellapneumoniae in Nagpur, central India. Indian J Med Res 1997; 105 : 158-61

[7]. Bauernfeind, A., H. Grimm, and S. Schweighart. 1990. A new plasmidiccefotaximase in a clinical isolate of Escherichia coli. Infection 18:294-298. [PubMed

[8]. Mackie \&McCartney Practical Medical Microbiology; 14th Edition; Elsevier Publications. Chapter 7 - Tests For Identification Of Bacteria, Pp 131 - 150.

[9]. Koneman`s color atlas and textbook of diagnostic microbiology; 6th edition

[10]. Clinical and Laboratory Standards Institute. 2005 guidelines by CLSI/NCCLS - CLSI informational supplement. Approved standard M100- S15 Wayne, PA; 2000;565

[11]. Sambrook and Russell editors. In Molecular Cloning: A Laboratory Manual 3 edvol 1; CSHI Press. New York. 2007; 1.3.2-1.3.4

[12]. Meeta Sharma Sharma M, Pathak S, Srivastava P. Prevalence and antibiogram of Extended Spectrum $\beta$-Lactamase (ESBL) producing Gram negative bacilli and further molecular characterization of ESBL producing Escherichia coli and Klebsiella spp. J Clin Diagn Res. 2013 Oct; $7(10): 2173-7$.

[13]. N FamFam, M. Diab, H. Helmi and I. El-Defrawy Phenotypic Detection of Metallo- $\beta$-Lactamases and Extended Spectrum $\beta$ Lactamases Among Gram Negative Bacterial Clinical Isolates Egyptian Journal of Medical Microbiology, October 2006 Vol. 15, N

[14]. NeemaShashwati, TripathiKiran and A.G.Dhanvijay Study of extended spectrum $\beta$-lactamase producing Enterobacteriaceae and antibiotic coresistance in a tertiary care teaching hospital J Nat SciBiol Med 2014 Jan-Jun;5(1) :30-35

[15]. Rodríguez-Bano J et al. Arch Intern Med. 2008;168:1897-902

[16]. R. Canton, A. Novais, A. Valverde, E. Machado, L. Peixe, F. Baquero and T. M. Coque. Prevalence and spread of extendedspectrum $\square$-lactamase -producing Enterobacteriaceae in Europe. CMI, 14 (Suppl. 1), 144-153 2008;26(4): 356-60.

[17]. Goossens H, Mystic Study Group MYSTIC program: summary of European data from 1997 to 2000.Diagn Microbiol Infect Dis. 2001;41(4):183-9. [PubMed]

[18]. Mathur P, Kapil A, Das B, Dhawan B. Prevalence of extendedspectrum $\beta$ - lactamase producing Gram negative bacteria in tertiary care hosptital. Indian J Med Res 2002;115:153-157.

[19]. MetriBasavaraj C., Jyothi P., PeerapurBasavaraj.V: The Prevalence of ESBL among Enterobacteriaceae in a Tertiary Care Hospital of North Karnataka, India ; Journal of Clinical and Diagnostic Research. 2011 June, Vol-5(3): 470-475.

[20]. Lautenbach E, Patel JB, Bilker WB, Edelstein PH, Fishman NO. Extended-spectrum $\beta$-lactamase-producing Escherichia coli and Klebsiellapneumoniae: Risk factors for infection and impact of resistance on outcomes. Clin Infect Dis. 2001;32:1162-71.

[21]. Kashyap G, Gupta S, Mamoria VP, Durlabhji P, Jain D. INCREASING prevalence of extended spectrum beta lactamases (esbls) producing e.coli and klebsiellaspp in outpatient departments (opds) patients in urinary tract infections (utis) in tertiary care hospital. IJCRR. (2013), [cited November 10, 2014]; 5(11): 80-86.

[22]. Nibedita Das et al. 10. Das N, Borthakur AK. Antibiotic coresistance among extended-spectrum beta lactamase- producing urinary isolates in a tertiary medical center: A prospective study. Chron Young Sci. 2012;1:53-6

[23]. Kiratisin P, Apisarnthanarak A, Laesripa C, and Saifon P. Molecular characterization and epidemiology of extended-spectrum $\beta$ lactamaseproducing Escherichia coli and Klebsiellapneumoniae isolates causing health care-associated infection in Thailand, where the CTX-M family is endemic. Antimicrob Agents Chemother 2008;52(8):2818-24.

[24]. Shakti Rath, DebasmitaDubey, Mahesh C. Sahu,etal.Surveillance of multidrug resistant Escherichia coli in a hospital in India.

[25]. Vemula S and Vadde R. Prevalence of ESBL-Producing Klebsiellapneumoniae Isolates in Tertiary Care Hospital. ISRN Microbiology. 2011; 2011: 318-48.

[26]. VarshaGupta Hena Rani, NidhiSingla, NeelamKaistha, and JagdishChanderDetermination of Extended-Spectrum $\beta$-Lactamases and AmpC Production in Uropathogenic Isolates of Escherichia coli and Susceptibility to FosfomycinJ Lab Physicians. 2013 JulDec; 5(2): 90-93

[27]. Ndugulile F, Jureen R, Harthug S, Urassa W, Langeland N. Extended spectrum beta-lactamases among Gram-negative bacteria of nosocomial origin from an intensive care unit of a tertiary health facility in Tanzania. BMC Infect Dis. 2005 Oct 15;5:86. 
[28]. Marra AR, Wey SB, Castelo A, Gales AC, Cal RGR, Filho JRC, et al. Nosocomial bloodstream infections caused by Klebsiellapneumoniae: impact of extended-spectrum $\beta$-lactamase (ESBL) production on clinical outcome in a hospital with high ESBL prevalence. BMC Infect Dis. 2006;6:24

[29]. Dasgupta Rubin, SapkotaRajendraPrevalance of Extended spectrum beta lactamase producing Escherichia coli and Klebsiella isolated from various inpatient department samples. IJRP $2012 ; 3(5): 428-31$.

[30]. ManinderKaur, ArunaAggarwalOccurance of the CTX-M, SHV and the TEM Genes Among the Extended Spectrum b-Lactamase Producing Isolates of Enterobacteriaceae in a Tertiary Care Hospital of North India Journal of Clinical and Diagnostic Research. 2013 April, Vol-7(4): 642-645

[31]. Gaurav D. Prevelance of Extended spectrum $\beta$-lactamase (ESBL) Producers among Gram Negative Bacilli from Various Clinical Isolates in a Tertiary Care Hospital at Jhalawar, Rajasthan, India. J ClinDiagn Res 2012;6:182-7.

[32]. R. Eshwar Singh, M. Veena, K.G. Raghukumar, G. Vishwanath, P.N. Sridhar Rao, B.V. MurlimanjuESBLresistace patterns in Escherichia coli and klebsiellapneumonia,astudt by DDST method International Journal of Applied Biology and Pharmaceutical Technology Volume: 2: Issue-4: Oct - Dec -2011.

[33]. PathakAPathak A, Marothi Y, Kekre V, Mahadik K, Macaden R, Lundborg CS. High prevalence of extended-spectrum betalactamase-producing pathogens: results of a surveillance study in two hospitals in Ujjain, India. Infect Drug Resist. 2012;5:65-73

[34]. Rudresh SM, NagarathnammaTExtended spectrum $\beta$-lactamase producing Enterobacteriaceae\&amp; antibiotic co-resistance.

[35]. Indian J Med Res. 2011 Jan; 133():116-8.

[36]. Kargar M,, Jahromi MZ, Najafi A, Ghorbani-Dalini S. Molecular detection of ESBLs production and antibiotic resistance patterns in Gram negative bacilli isolated from urinary tract infections. Indian J Pathol Microbiol. 2014 Apr-Jun;57(2):244-8.

[37]. VidyaPai, Sunil Rao P, Bhaskaran Nair Multiple $\beta$ lactamase enzymes producing clinical isolates of gram negative Bacteria in a teaching hospital Int J Pharm Bio Sci 2013; 3(1) 590-

[38]. Choi SH1, LeeJE, ParkSJ.KimMN,ChooEJ,KwakYG, Jeong JY, WooJH, KimNJ, KimYS Prevalence, microbiology, and clinical characteristics of extended-spectrum beta-lactamase-producing Enterobacter spp., Serratiamarcescens, Citrobacterfreundii, and Morganellamorganii in KoreaEur J ClinMicrobiol Infect Dis 2007 Aug:26(8):557-61.

[39]. Shrestha $S^{1}$ AMatya R, Datta R Prevalence of extended spectrum beta lactamase (ESBL) production in gram negative isolates from pyogenic infection in tertiary care hospital of eastern Nepal.Nepal Medical Coll J. 2011Sep;13(3):186-9

[40]. Emery CL, Weymouth LAJ ClinMicrobiol. 1997 Aug; 35(8):2061-7.[PubMed] [Ref list]Detection and clinical significance of extended-spectrum beta-lactamases in a tertiary-care medical center

[41]. Manoharan A, Premalatha K, Chatterjee S, Mathai D. Correlation of TEM, SHV and CTX-M extended spectrum beta-lactamases among Enterobacteriaceae with their in vitro antimicrobial susceptibility. Indian J Med Microbiol. 2011;29(2):161-64.

[42]. SanjeevkumarSanjeev Kumar, Sudhir Kumar Mehra,R.C.Kanta Extended Spectrum B-Lactamases among Clinical Isolates of Enterobacteriaceae Ssp.: Prevalence and Susceptibility Pattern at A Tertiary Care Hospital Sch. J. App. Med. Sci., 2014; 2(2D):862-864.

[43]. Akbar M Rafey, Al-Muharrmi Z, Toki R Prevalence of extended-spectrum beta-lactamases-producing isolates over a 1-year period at a University Hospital in Oman.Saudi Med J. 2007 Jan; 28(1):22-7.

[44]. R. Bonnet Growing group of extended-spectrum $\beta$-lactamases: the CTX-M enzymes," Antimicrobial Agents and Chemotherapy, vol. 48, no. 1, pp. 1-14, 2004

[45]. Wong-BeringerA Therapeutic challenges associated with extended-spectrum, beta-lactamase-producing Escherichia coli and Klebsiellapneumoniae.Pharmacotherapy. 2001 May; 21(5):583-92..

[46]. Ullah F, Malik SA, Ahmed J. Antibiotic susceptibility pattern and ESBL prevalence in nosocomial Escherichia coli from urinary tract infections in Pakistan.Afr J Biotechnol. 2009;8:3921-6.

[47]. Jacoby GA, Mediros AA. More extended-spectrum Blactamases.Antimicrob Agents Chemother 1991; 35:1697-704

[48]. Martínez-Martínez L, Pascual A, ConejoMdel C, García I, Joyanes P, Doménech-Sánchez A, Benedí VJ Antimicrob Agents Chemother. 2002 Dec; 46(12):3926-32

[49]. Kaftandzieva A, Trajkovska-Dokic E, Panovski N. Prevalence and molecularcharacterization of Extended Spectrum BetaLactamases (ESBLs) producingEscherichia Coli and Klebsiella Pneumoniae. Prilozi. 2011 Dec;32(2):129-41.

[50]. Ho, P. L., W. W. Poon, S. L. Loke, M. S. Leung, K. H. Chow, R. C. Wong, K. S. Yip, E. L. Lai, and K. W. Tsang. 2007. Community emergence of CTX-M type extended-spectrum -lactamases among urinary Escherichia coli from women. J. Antimicrob. Chemother. 60:140-144

[51]. Feizabadi MM ${ }^{1}$, Delfani S, Raji N, Majnooni A, Aligholi M, Shahcheraghi F, Parvin M, Yadegarinia D. Distribution of bla(TEM), bla(SHV), bla(CTX-M) genes among clinical isolates of Klebsiellapneumoniae at Labbafinejad Hospital, Tehran, Iran.Microb Drug Resist. 2010 Mar;16(1):49-53.

[52]. Mubarak SA, Abida AE, Hajar MA, Adeel IA. Molecular Characterization and Epidemiology of Extended-Spectrum BetaLactamase-Producing Escherichia coli and Klebsiellapneumoniae Isolates in the United Arab Emirates. Med PrincPract. 2011;20:177-80.

[53]. James H. Jorgensen,1 * M. L. McElmeel,1 L. C. Fulcher,1 and B. L. Zimmer2 Detection of CTX-M-Type Extended-Spectrum BetaLactamase (ESBLs) by Testing with MicroScan Overnight and ESBL Confirmation Panels

[54]. Livermore DM, Canton R, Gniadkowski M, Nordmann P, Rossolini GM, Arlet G, et al. CTX-M: changing the face of ESBLs in Europe. J AntimicrobChemother. 2007 Feb;59:165-74. [PubMed]

[55]. Bonnet R. Growing group of extended-spectrum beta-lactamases: the CTX-M enzymes. Antimicrob Agents Chemother. 2004;48:114. [PMC free article] [PubMed]

[56]. MathaiD,ManoharanA, VAsanthan G Epidemiology and implications of ESBL(2009)Crit Care Update 2009; 14:152-62 